\title{
ESTUDIO PRELIMINAR DEL ZOOPLANCTON DE LAS SALINAS DE CADIZ
}

\author{
M. Yúfera, L.M. Lubián y E. Pascua 1 \\ Instituto de Investigaciones Pesqueras de Cádiz (C.S.I.C)
}

Palabras Clave: Hypersaline ecosystem, zooplankton of the SW Spain

\author{
A B S TR ACT \\ PRELIMINARY STUDY ON ZOOPLANKTON IN SALT-MARSHES OF CADIZ (SPAIN)
}

The evolution of the main planktonic groups of salt-marshes in Cádiz area (SW Spain) has been determined. Collection were made during two years. From spring to autumn the pond gates remain closed and the water renovation is partial and occasional. In this time, the zooplankton composition is different from that found immediately out-gates, and the most zooplankters are euryhaline species. Out-gates there are abundance of tintinnids, primarily Tintinopsis, rotifers of the genus Synchaeta, Balanus nauplü, and presence of calanoids among the copepods. The samples of fish-ponds present harpacticoids and cyclopoids, other species of rotifers as Brachionus plicatilis and Trichocerca spp. and an important population of the ciliate Fabrea salina.

The plankton communities are influenced by management, which controls the changes in salinity and organic matter accumulation in each salt-marsh.

\section{INTRODUCCION}

En los últimos años las salinas de la riberagaditana han adquirido una creciente atención debido a su potencial interés para el desarrollo de cultivos marinos. En ésta línea, se han realizado algunos trabajos sobre las condiciones del medio (Arias y Establier, 1979; Establier et al., 1984) y biología y producción de algunas especies de peces (Arias et al., 1980) que muestran resultados variables de unas salinas a otras y que apuntan hacia una variabilidad en la cantidad de presas disponibles determinadas por ciertos condicionamientos ambientales, principalmente hidrológicos y humanos. En este contexto, el conocimiento de las comunidades planctónicas tiene un doble interés por su cualidad de organismos indicadores en un habitat intermareal, y por constituir parte del alimento directo del alevinaje que anualmente admite cada salina y de las presas que mantendrán su engorde.

Este trabajo muestra los resultados obtenidos en dos años de muestreo en tres salinas de la zona, describiéndose las características del zooplancton obtenido. Igualmente se incluyen algunos animales bentónicos captados con el muestreo realizado. De esta forma, se establece un conocimiento previo para diseñar trabajos con mayor profundidad.

\section{DESCRIPCION DEL MEDIO}

Las salinas de Cádiz (S.O.de España) abarca una extensión aproximada de $8.000 \mathrm{Ha}$, y están situadas en un terreno arcilloso rellenado durante el pleistoceno por los aportes del río Guadalete. Cada salina está constituida por una serie de lagunas de extensión y forma variables, con una profundidad máxima de 1 a 2 metros en el estero (laguna principal de captación de agua y cría de peces) y una mínima de unos pocos cms en los cristalizadores de sal. Los esteros están separados con una compuerta con una red de caños que comunican con la bahía de Cádiz y el Océano Atlántico. El clima es mediterraneo con temperaturas 
máximas de $30-35^{\circ} \mathrm{C}$ en verano, y mínima de $6-12^{\circ} \mathrm{C}$ en invierno. El único aporte importante de agua dulce lo constituyen las lluvias, que durante los años de muestreo han sido muy escasas debido a una prolongada sequía. La caracteristica más importante es el régimen de vientos, que son persistentes, mostrando una alternancia este(seco)-oeste(humedo) y que inciden directamente sobre la salinidad del agua de la zona, principalmente en los meses de verano y otoño.

El régimen hidrológico es consecuencia de la explotación conjunta de sal y peces en los últimos siglos, aunque actualmente la extracción de sal está abandonada en la mayoría de las salinas. Durante los meses de invierno y hasta el inicio de la primavera las compuertas estan completamente abiertas, y las

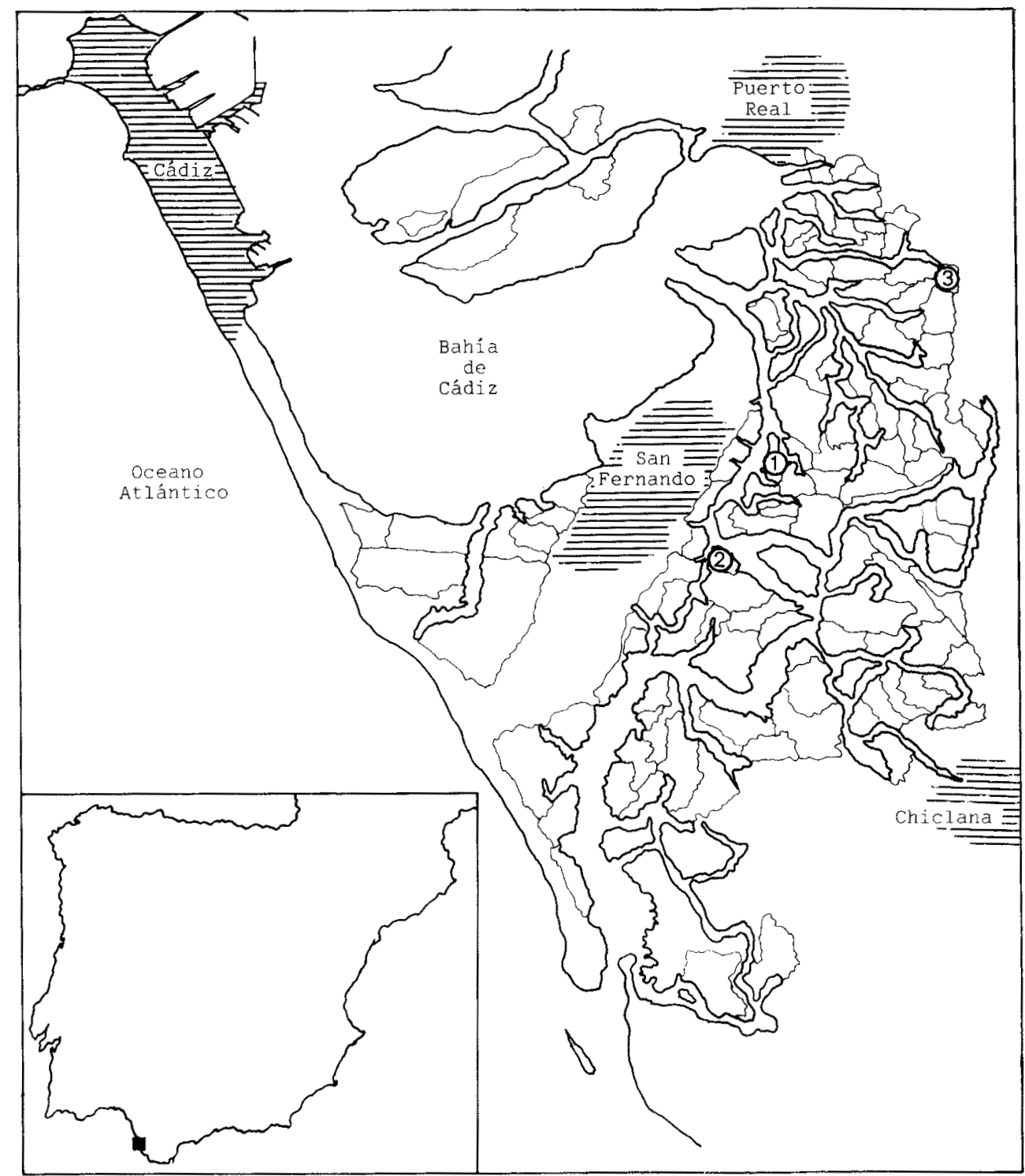

Figura 1 - Situación de las salinas estudiadas 1 i Salina de "Nuestra Señora de la $O^{\prime 2}$ 2) salina de "San Agapito". 3) Salina de "San Rafael del Monte"

Maps showing location of salt-marshes studied: 1) "Nuestra Señora de la 0 " salt-marsh 2) "San Agapito" salt-marsh. 3) "San Rafael del Monte" salt-marsh 
salinas se llenan y vacian con las mareas. Posteriormente las compuertas se cierran y el estero queda completamente lleno, realizándose sólo una renovación parcial y esporádica del agua para intentar mejorar la oxigenación.

Los fondos y superficies del agua estan ocupados por masas de algas macrófitas, a menudo desprendidas del sustrato y variando su posición según los vientos. Por otra parte, las tomas de marea levantan gran cantidad de detritus y material particulado del sedimento que puede quedar, a veces de forma muy abundante, en suspensión.

\section{MATERIAL Y METODOS}

Se han estudiado las muestras recogidas entre abril de 1981 y abril de 1983 en las salinas de "Nuestra Señora de la O", "San Agapito" y "San Rafael del Monte" (figura 1). En cada una se han realizado dos puntos de muestreo, uno en el interior del estero, y otro en el caño de alimentación, ambas junto a las compuertas de toma de agua. Durante el periodo en que las compuertas permanecieron abiertas sólo se muestreó en el interior del estero. Las muestras se tomaron en superficie, con una periodicidad de una o dos veces por mes. El zooplancton se recogió filtrando de 60 a 120 litros de agua, recogidos con una garrafa, a través de un filtro de $25 \mathrm{pm}$ de luz de malla, y posteriomente se fijó con formol al $4 \%$. Conjuntamente se midió la temperatura y la salinidad de la muestra (tomada a primeras horas de la mañana).

\section{RESULTADOS}

La salina de "Nuestra Señora de la O" está situada junto al caño de Sancti Petri, brazo de mar que comunica la bahía con el océano, y está cercana a los efluentes urbanos de San Fernando. La salinidad del caño en este punto se mantiene entre 36 y 38 g. por 1 ., y sólo desciende cuando ha habido temporales de lluvia. La temperatura, que es similar para las tres salinas, oscila entre unos valores de $23-26^{\circ} \mathrm{C}$ en el periodo estival y 10-12QCen invierno, tanto en el caño como en el estero (figura 2). Esta salina presenta gran acumulación de masas de algas y materia orgánica en descomposición por tener mas abandonado el mantenimiento y la limpieza de fondos.
Las muestras de este estero presentan mayor abundancia de zooplancton, principalmente en los grupos que incluyen los organismos de menor talla (ciliados, rotíferos y velígeras de moluscos). En el caño, los ciliados están representados por tintínidos (principalmente del género Tintinnopsis), que muestran las mayores densidades durante los meses de verano y otoño. En el estero, el organismo más abundante es Fabrea Salina, ciliado heterotrico de 50500 um que llega a ser la especie dominante durante gran parte del año. La población de Fabrea se reduce sensiblemente, casi desaparece, cuando son abiertas las compuertas de la salina en otoño, volviendo a aparecer cuando la salinidad comienza a ascender tras el cierre de las compuertas en primavera.

Synchaeta spp. es el rotífero más constante en el caño, con densidades que escilan entre 1 y 20 ind. por litro, aunque pueden aparecer en altas densidades. Se encuentra también en el estero mientras la salinidad no se haya incrementado excesivamente. El organismo más característico de este grupo es Bracbionus plicatilis, que forma una población típica de verano a otoño en el interior del estero. Alcanza densidades cercanas a los 400 individuos por litro, no encontrándose el resto del año, puesto que desaparece totalmente cuando se abren las compuertas.

Las velígeras de bivalvos son las más abundantes entre las larvas de moluscos. Aparecen durante codo el año en el caño y en el estero en el periodo en que las compuertas están abiertas. Las mayores densidades en el caño se han encontrado en el mes de junio (200400 individuos por litro), siendo las más bajas durante los meses de invierno. Tambien llega a ser muy abundante en el interior del estero cuando está cerrado (hasta 100 ind. por 1.), pero desaparece en verano coincidiendo con los valores más altos de salinidad. Las velígeras de gasterópodos son mucho menos abundantes, aparecen permanentemente en las muestras del caño, oscilando entre 1 y 20 ind./l. Sólo en los primeros meses de 1983 han superado los 50 ind/1. En el estero aparecen cuando está abierto, y muy raramente cuando está cerrado.

Dentro de los copépodos, los estadios de desarrollo que normalmente aparecen son los nauplius y copepoditos. Los adultos son escasos, debido a que pertenecen principalmente a especies bentónicas que viven en el sedimento y en las masas de macrófitos. El interior del estero ha presentado mayor abundancia de nauplius que el caño, alcanzándose a veces densidades muy altas, cercanas a los 1300 ind/1. Los 

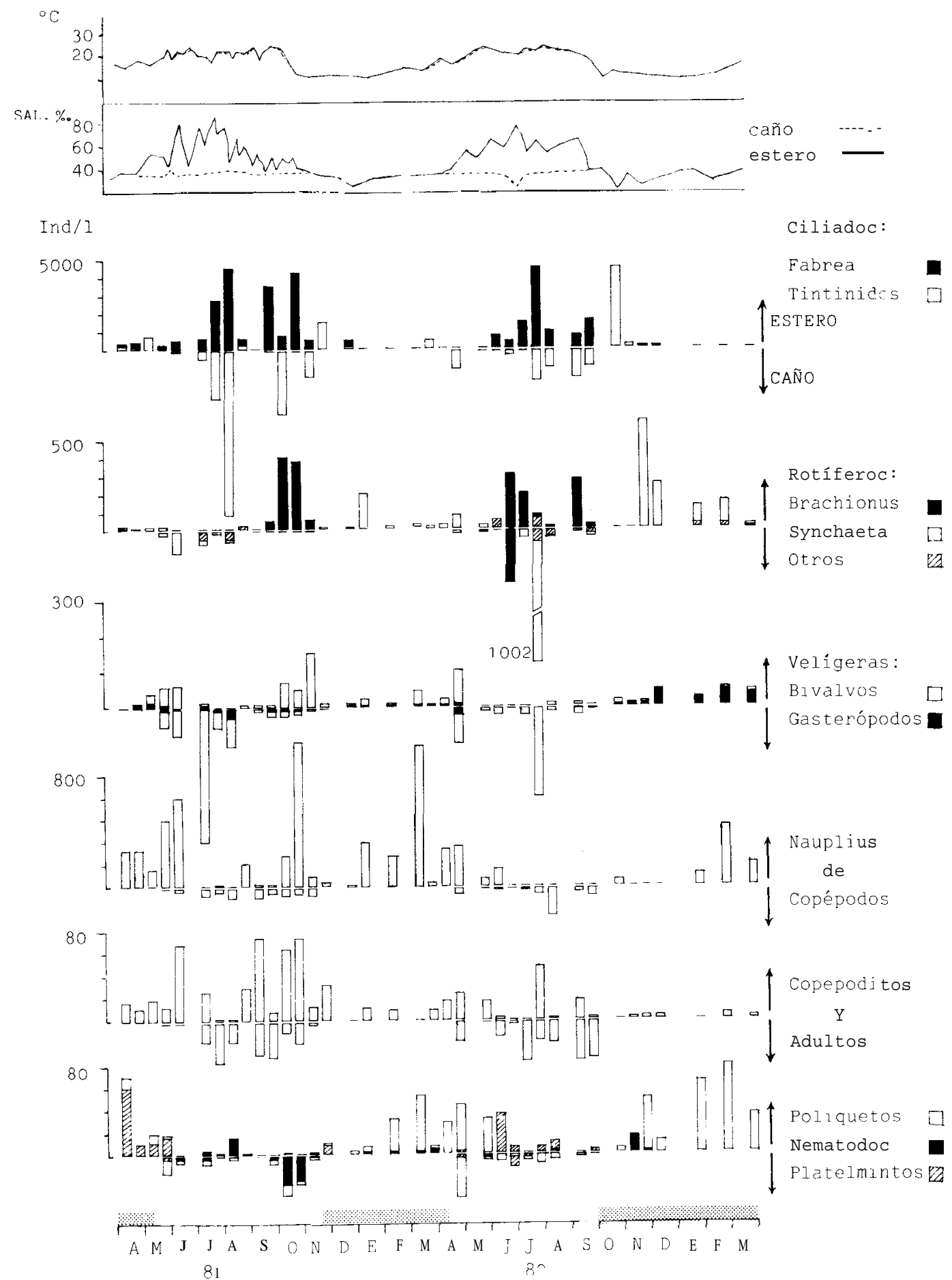

Figura 2.- Temperatura, salinidady densidad de los diterentes grupos zooplantónicosen las salinas de "Nuestra Señora de la O". Las barras verticales indican el número total de individuos en cada muestra. Las barras horizontales muestran el periodo en que las compuertas estan permanentemente abiertas

Temperature, salinity and density of different zooplanktonic groups in "Nuestra Señora de la $\mathrm{O}$ " salt-marsh. Vertical bars indicate the toral number of individuals in each sample. Horizontal bars show the time during pond-gates remain opened. 
copepoditos y adultos son más abundantes desde primavera a otoño, tanto en el caño como en el estero. Generalmente existe una predominancia de ciclopoides y harpacticoides, aunque en ocasiones aparecen grandes densidades de calanoides con una destacada presencia de Acartia grani, que es la especie mas usual en la zona de la bahía de Cádiz.

Las larvas de poliquetos aparecen normalmente en el caño ( 1 a 10 ind/l) y en el estero cuando está abierto. época en la que alcanzan las mayores densidades (6080 ind / 1 ). A veces, también se encuentran en el estero cuando está cerrado en los meses de primavera. los nematodos aparecen irregularmente a lo largo de todo el año y los platelmintos sólamente durante los meses de primavera-verano.

En esta salina, a finales de verano y otoño, se observa una comunidad constituída por Fabrea salina, Brachionus plicatilis y copépodos, principalmente nauplius, que probablemente está relacionada con la densa población de la eustigmatofíea Nannochloropsis spp. que se desarrolla en este estero a partir del cierre de las compuertas (Lubian et al, en - preparación).

La salina de "San Agapito" toma tambien el agua directamente del caño Sancti Petri, pero está situada más hacia la desembocadura del océano Atlántico. La evolución anual de los valores de temperatura y salinidad es similar a la expuesta en la salina de "N.S. de la O", pero no se alcanzan salinidades tan elevadas, permaneciendo alrededor de los $60 \mathrm{~g} / 1$ durante el verano (figura 3 ).

Entre los ciliados sólo aparecen los tintínidos, que están presentes en las muestras del caño y del estero cuando está abierto. Como en la salina anterior forman poblaciones de verano y otoño.

Los rotíferos son mucho más escasos que en la salina de "N.S. de la $\boldsymbol{O}$ ". El más corriente es Synchaeta spp., que también en este caso se presenta en el caño, y sólo aparece en el estero cuando la salinidad no es muy alta. Existe además una población estival de Trichocerca spp. que en el primer año de estudio alcanzó densidades superiores a 350 ind/l.

Las velígeras de moluscos presentan la misma evolución que la referida en la salina anterior, con una coincidencia en los máximos de larvas de bivalvos en las muestras de aguas sujetas al régimen mareal. Las velígeras de gasterópodos no superan los 15 ind/l.

Las menores frecuencias de copépodos, tanto en el caño como en el estero se dan en los meses en que las compuertas están abiertas. La diferencia más apreciable con la salina anterior es la ausencia de un máximo de abundancia de nauplius en las muestras de principio de 1982.

En esta salina las larvas de poliquetos y los nematodos son menos abundantes. Igualmente sucede con los platelmintos, excepto en un pico de abundancia en primavera de 1981 .

La salina de "San Rafael del Monte" es la que ha presentado menor cantidad de plancton (fig. 4). La salinidad del caño es más elevada que en las otras dos, alcanzándose en ocasiones valores superiores a los 50 $\mathrm{g} / \mathrm{l}$, esto es debido a su situación en fondo de saco con menor renovación de agua que el caño de Sancti Petri. Por el contrario, las salinidades medidas en el estero son algo inferiores, tal vez debido a que la profundidad de este estero es algo mayor.

La presencia de tintínidos en el caño es muy escasa, sólo en una muestra llega a 370 ind/1. con similar abundancia aparece Fabrea salina, siendo menor su presencia en el interior del estero.

Entre los rotíferos sólo Synchaeta spp alcanza densidades apreciables, apareciendo unicamente en el caño. Sin embargo, las velígeras de moluscos son más abundantes en el estero. La evolución y abundancia de las poblaciones de copépodos es similar a "San Agapito".

En el último grupo representado (larvas de poliquetos, nematodos y platelmintos), la diferencia más apreciable es la ausencia de los picos de alta frecuencia de nematodos encontrados en la primavera de 1981 en las otras dos salinas.

Entre los organismos que aparecen ocasionalmente en las tres salinas caben destacar los nauplius de cirrípedos, muy abundantes en la bahía, así como ostrácodos, anfípodos, larvas de quironómidos y coleópteros precedentes de las masas de macrófitos.

\section{DISCUSION}

Independientemente de la diferencia de abundancia que se observa entre cada salina, la variedad de organismos es bastante pobre, y está representado por algunas especies planctónicas, fases larvarias de otros organismos y algunas especies bentónicas que, dadas la poca profundidad y la turbulencia del ambiente intermareal estudiado, se presentan asiduamente y con frecuencia irregular en las muestras.

La mayor abundancia de plancton encontrado en "N. S. de la O" puede ser debida a su mayor 


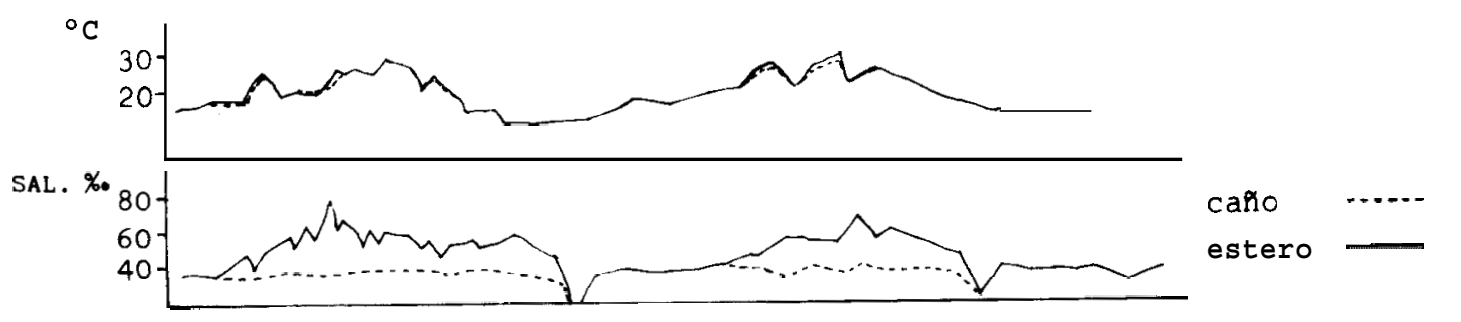

Ind $/ 1$
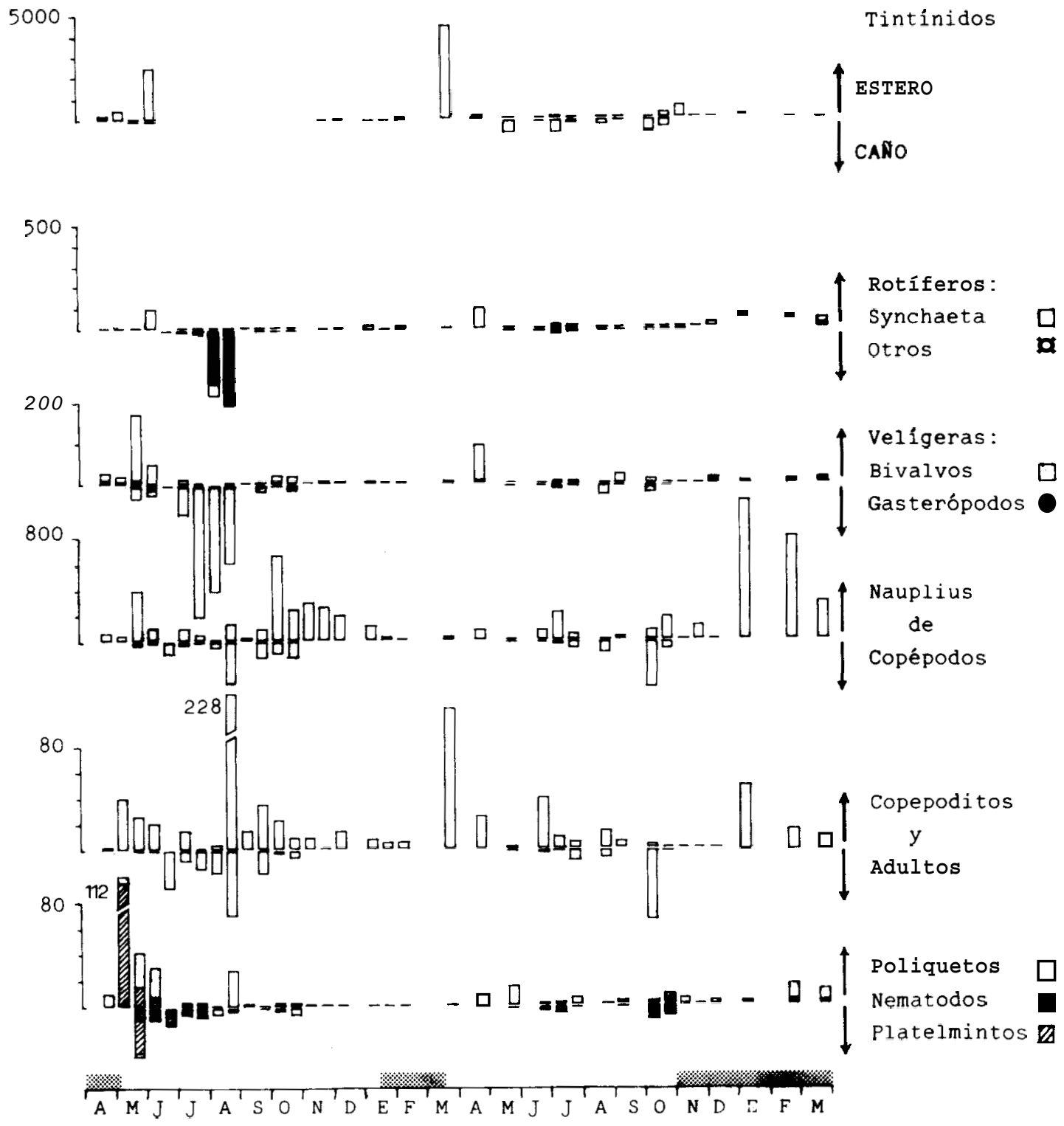

Figura 3 - Temperatura,salinidad y densidad de los diferentes grupos zooplanctóni ous de la salina de "San Agapito". Leyenda como en la ${ }^{\text {figura }} 2$. Temperature, salinity and density of zooplanktonic groups in "San Agapito" salt-marsh. Symbols as figure. 2. 

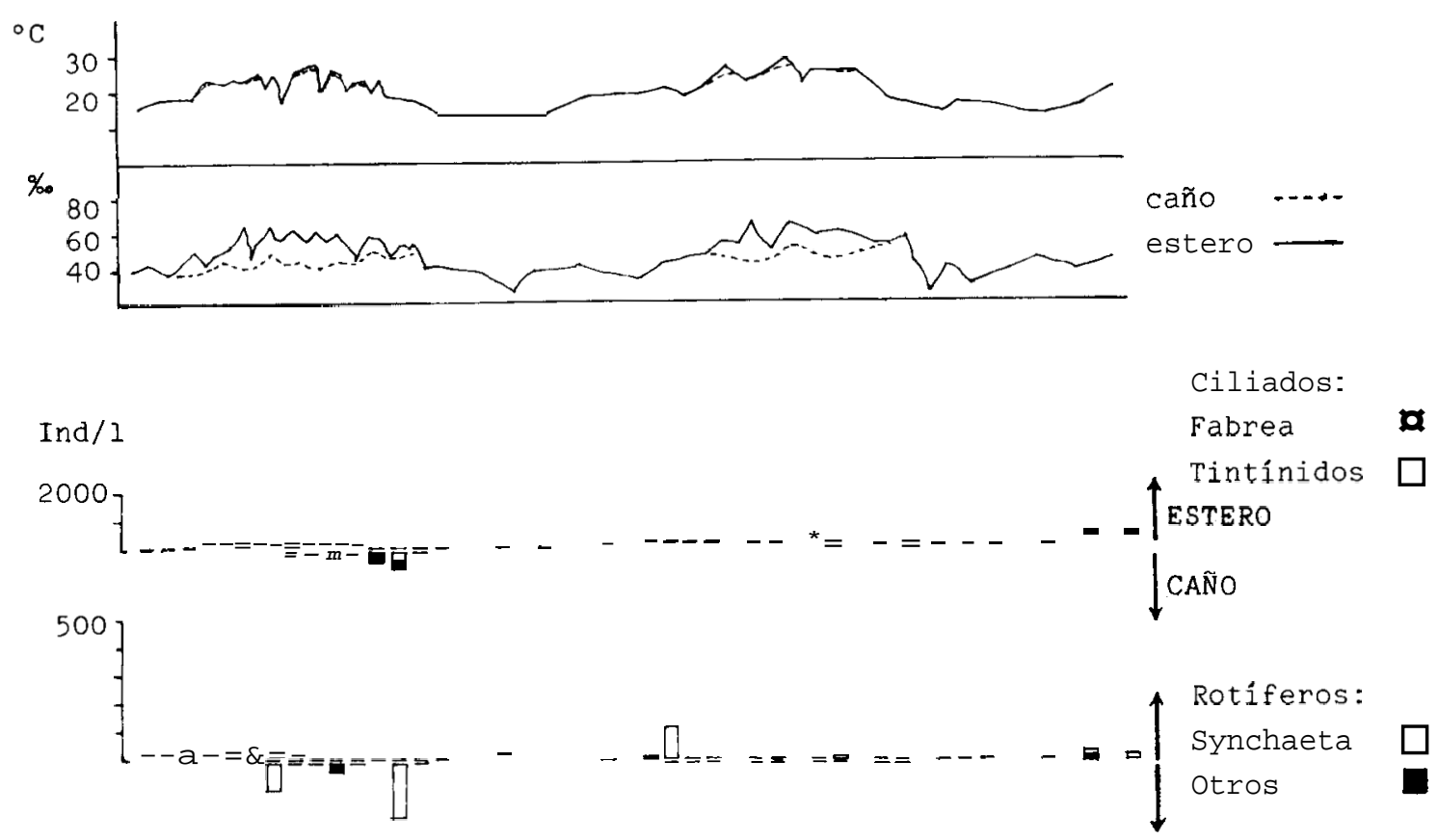

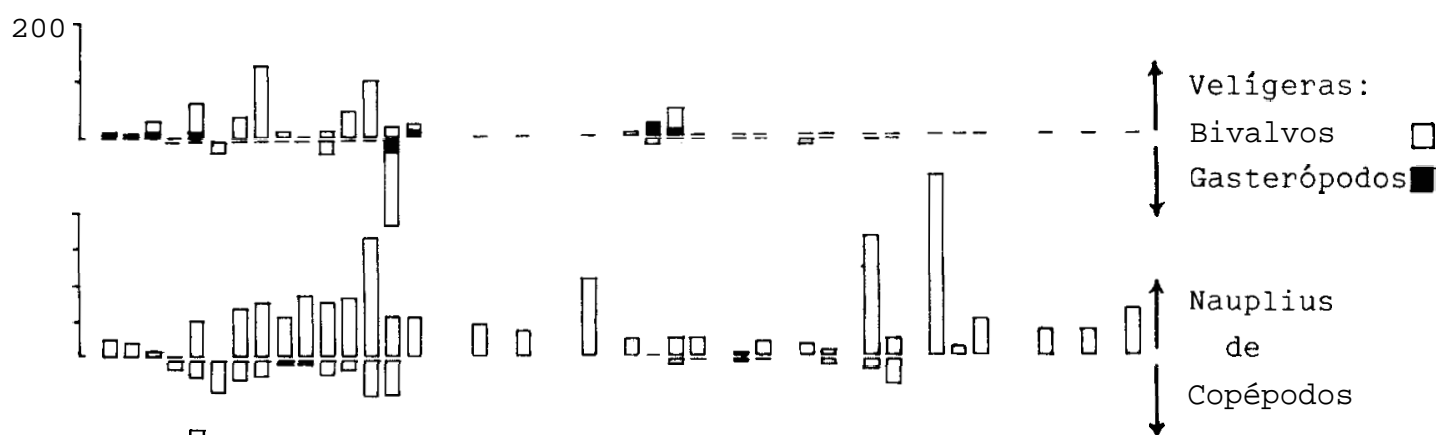

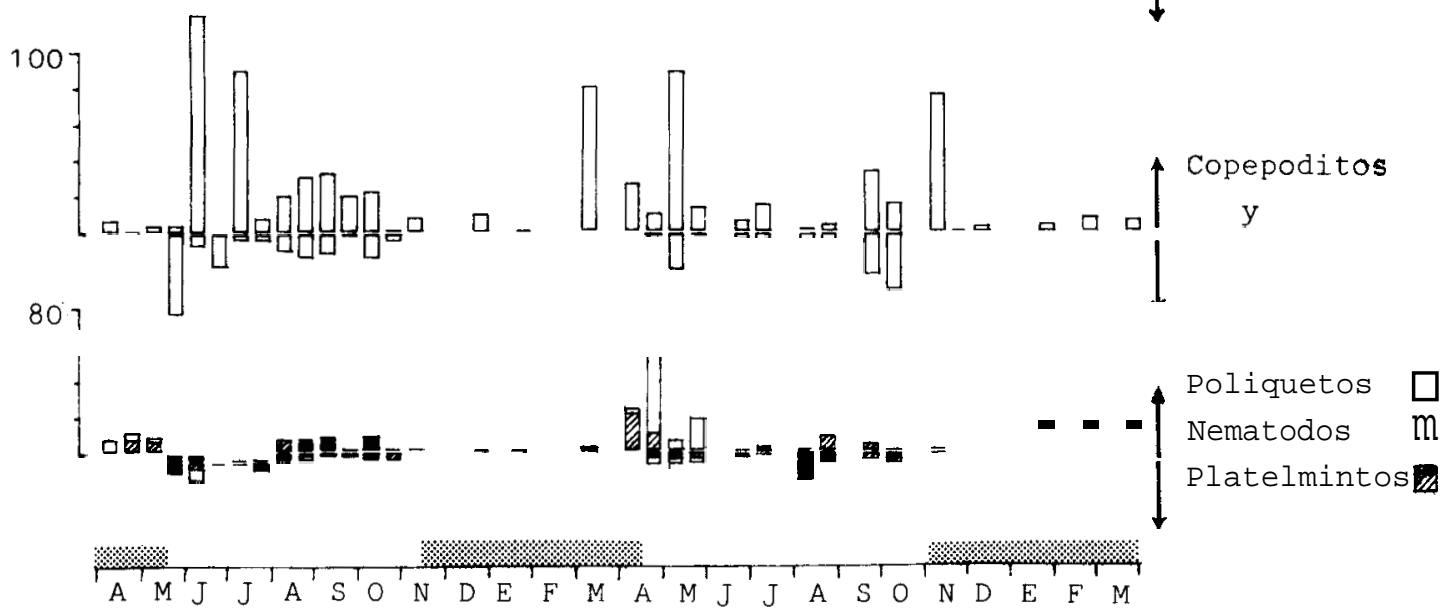

Figura 4.- Temperatura, salinidad y densidad de los diferentes grupos zooplanctónicos en la salina de "San Rafael del Monte".Leyenda como en la figura 2 .

Temperature, salinity and density of different zooplanktonic groups in "San Rafael del Monte" salt-marsh. Symbols as figure 2 
proximidad a los efluentes urbanos de la San Fernando y a la mayor presencia de fitoplancton y materia orgánica (Lubian et al., en preparación). Por otra parte, se observa en las tres salinas una menor cantidad de zooplancton en invierno, hecho atribuíble no sólo al desarrollo de poblaciones estivales, como los tintínidos y larvas de bivalvos, sino tambien a la presencia de poblaciones típicas de ambientes de salinidad variable y menos renovados, comoFabrea salina (De Winter y Persoone, 1976), Brachionus plicatilis (Pascual y Yúfera, 1983) y copépodos harpacticoides y ciclopoides. Aunque se observan organismos asiduos en el caño y organismo típicos del interior de las salinas, existe una influencia conjunta entre ambos, debido a la renovación parcial y ocasional de agua en todas las salinas. De esta forma podemos encontrar Fabrea y Brachionus en el caño, y tintínidos en los esteros se ha habido un intercambio de agua previo a la toma de la muestra. Esta influencia hace que los caños, aunque directamente comunicados con la bahía y el océano, presenten ciertas diferencias con respecto a ellos. En comparación con la bahía no se han encontrado los característicos "blooms" invernales de cladóceros, ni la abundancia de calanoides.

Los factores que más influyen en la evolución de las comunidades del interior de los esteros es la salinidad y la cantidad de fitoplancton y materia orgánica acumulada, que están ligados a la renovación de agua, y que depende directamente del criterio del capataz y del grado de limpieza de fondos a que se haya sometido cada salina. Los valores medios que alcance la salinidad van a determinar la selección de ciertas especies, y además los cambios bruscos y esporádicos alteran el desarrollo de las poblaciones de especies eurihalinas.

Hay que tener en cuenta la limitación que suponen los métodos de muestreo para conocer el plancton de la zona. Por una parte, sólo se ha muestreado en los esteros y caños, no estudiandose otras zonas con menor o ninguna renovación, como son los cristalizadores de sal, donde abundan densas poblaciones del crustáceo Artemia salina. Además no son considerados otros organismos, como las larvas de decápodos que no son capturados con el sistema empleado, aunque están presentes normalmente en los muestreos realizados por arrastre de una manga de plancton. Tampoco se han considerado los misidáceos, organismos difíciles de recoger en los muestreos de plancton (Mauchline, 1980) que son muy abundantes a pocos $\mathrm{cms}$ del sedimento y que forman parte de la dieta de mugílidos y decápodos (Drake, 1973; Rodriguez, 1978).

\section{BIBLIOGRAFIA}

Arias, A. y Establier, R. 1979. Datos sobre las condiciones fisicoquimicas y el fitoplancton de los esteros de Cádiz. Inf. Técn Inst. Inv.Pesq., 70

Arias, A. Rodriguez, R.B. y Drake, P. 1980. Fish production in extensive polyculture on the esteros of Cadiz (Southern Spain). ICES Doc C.M 1980/F:6, Mariculture committe, 9p.

De Winter, F. y Persoone, G. 1976. Preliminary experiments with the ciliate Fabrea ralina as a potencial live food for mariculture purposes. In: Proc. 10th Europeam Symposium on Marine Biology Ostend, Belgium (G. Persoone an E. jasper ed.) vol 1: 17-48 Universa press, Watteren Belgium

Drake, P. 1983. Biología de los mugilidos en los esteros de las salinas de San Fernando (Cádiz) Niveles de producción extensiva, crecimiento y relaciones tróficas. Tésis doctoral, Universidad de Sevilla

Establier, R., Lubián, L.M., Blasco, J. y Gómez, A. 1984. Estudio de las variaciones fisicuquímicas de las salinas de Cádiz dedicadas al cultivo extensivo de peces. Inf. Técn. Inst. Inv. Pesq. 112.

Mauchline, J. 1980. Advances in marine biology, vol 18, The biology of mysids and euphausiids. (J.H.S. Blaxter; Sir F.S. Rusell and sir F. Yonge eds.), Academic press, Jondon, New York.

Pascual, E. y Yúfera, M. 1983. Crecimiento en cultivo de una cepa de Brachionus plicatilis O.F. Müller en función de la temperatura y la salinidad. Inv. Pesq, 47(1): 151-159.

Rodríguez, A. 1978. Consideraoones sobre la biología y posibilidades de cultivo del langostino, Penaeus kerathurus. del golfo de Cádiz. En Cultivos marinos en la provincia de Cádiz. Publ. del Banco Urquijo, Sevilla. 